\title{
Congenital Diaphragmatic Hernia: A Case Report On Bochdalek Type
}

\author{
S. Nayak ${ }^{1}$, S.P. Dash ${ }^{2}$, M.K. Khatua ${ }^{3}$ \\ ${ }^{1}$ Tutor, Department of Anatomy, SCB Medical College, Cuttack, Odisha, India \\ ${ }^{2}$ Consultant Radiologist, Sahara Diagnostics, Courtpeta Square, Berhampur, Odisha, India \\ ${ }^{3}$ Assistant Surgeon, DHH Koraput, Odisha, India
}

\begin{abstract}
Congenital Diaphragmatic Hernia $(\mathrm{CDH})$ is defined as the herniation of abdominal contents through an orifice in the diaphragm. Most common type of $\mathrm{CDH}$ is the Bochdalek hernia; other types include Morgagni hernia, posterior hernia and Central hernia of the diaphragm. Defect in the diaphragm allows the abdominal organs to push into the proper lung formation leading to hypoplastic lungs and abnormal vessels that cause respiratory insufficiency and persistent pulmonary hypertension with high mortality. The incidence is 1 in 2,500 live-births. This case report is about a full term male baby who died of respiratory distress within a few hours of birth. On dissection, a left-sided posterolateral diaphragmatic hernia with associated intestinal malrotation was found, suggestive of Bochdalek's hernia.
\end{abstract}

Keywords: Dextrocardia, Diaphragmatic hernia, Mediastinal shift, Pulmonary hypoplasia

\section{Introduction:}

The diaphragm is a dome-shaped musculotendinous partition that separates the pericardial and pleural cavities above it from the peritoneal cavity lying below it. Congenital Diaphragmatic Hernia $(\mathrm{CDH})$ is the herniation of abdominal contents into the thorax due to a defect in the diaphragm. The incidence is 1 in 2,500 live births and accounts for $8 \%$ of all major congenital anomalies ${ }^{1}$. It usually presents with severe respiratory distress in the neonatal period but sometimes the defect is not manifested until late childhood or even in adult life $^{2}$.

$\mathrm{CDH}$ occurs due to defective development of pleuroperitoneal membrane or failure of fusion of pleuroperitoneal membrane with other elements of the diaphragm. When the abdominal contents like intestines, stomach and/or spleen herniate in the thorax, they compress the developing lungs and cause their hypoplasia. $\mathrm{CDH}$ is more common on the left side probably because right pleuroperitoneal canal closes earlier than the left one. Very rarely $\mathrm{CDH}$ occurs bilaterally ${ }^{3}$.

\section{Case Report:}

A 36 years old pregnant woman came to Sahara Diagnostics, Berhampur from a nearby village for prenatal ultrasound at $30^{\text {th }}$ week of gestation where the baby was diagnosed with CDH (Fig. 1). A full term male baby weighing 2,700 grams was delivered vaginally on the Expected Date of Delivery after spontaneous labour. The baby developed severe respiratory distress within one hour and finally succumbed to death despite intensive resuscitation. With the parents' written consent, the dead baby was received, injected and immersed in routine formalin for fixation.

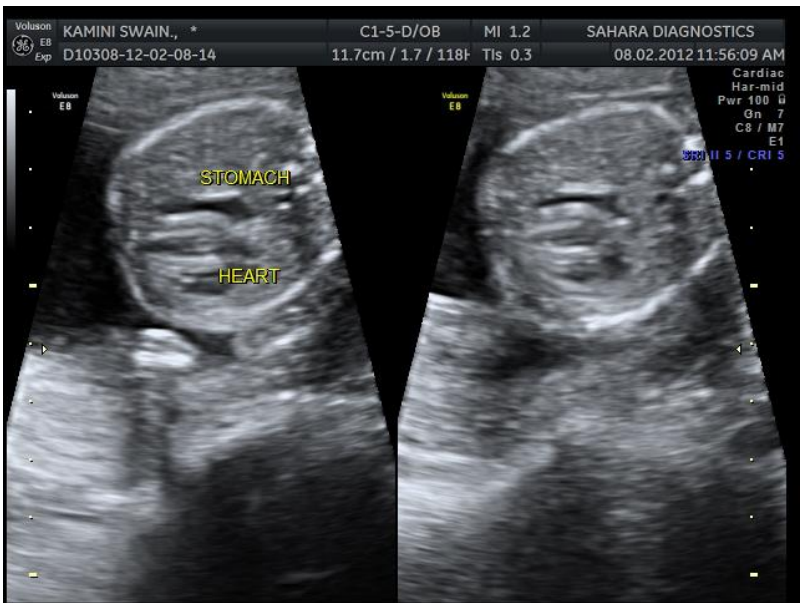

Figure 1: USG picture showing the stomach and heart in the thoracic cavity. 


\section{Observation:}

The chest and abdominal wall of the baby was incised and opened carefully. The chest cavity showed mediastinal shift towards right side. A large defect was found on the left side of the diaphragm, confirming $\mathrm{CDH}$. Whole of the gastrointestinal tract including a part of the liver were herniated to the left side of the pleural cavity. Only the large intestine, part of the liver and the urinary organs were seen in the abdominal cavity. The spleen was completely absent. There was severe hypoplasia of both the lungs and dextrocardia (Fig. 2 \&3).

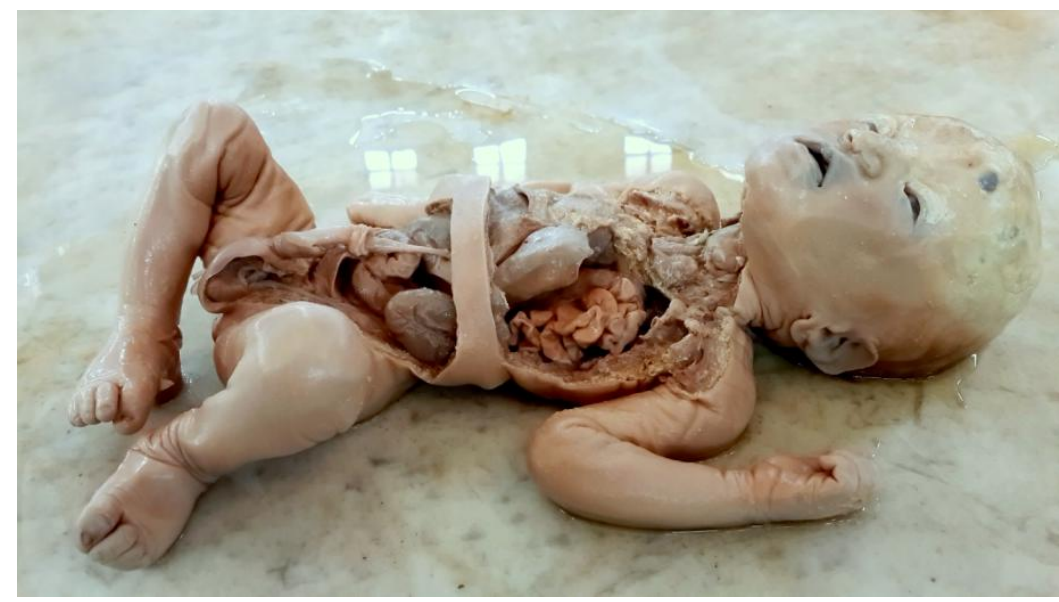

Figure 2 showing the dissection of thoracic and abdominal cavity with the CDH

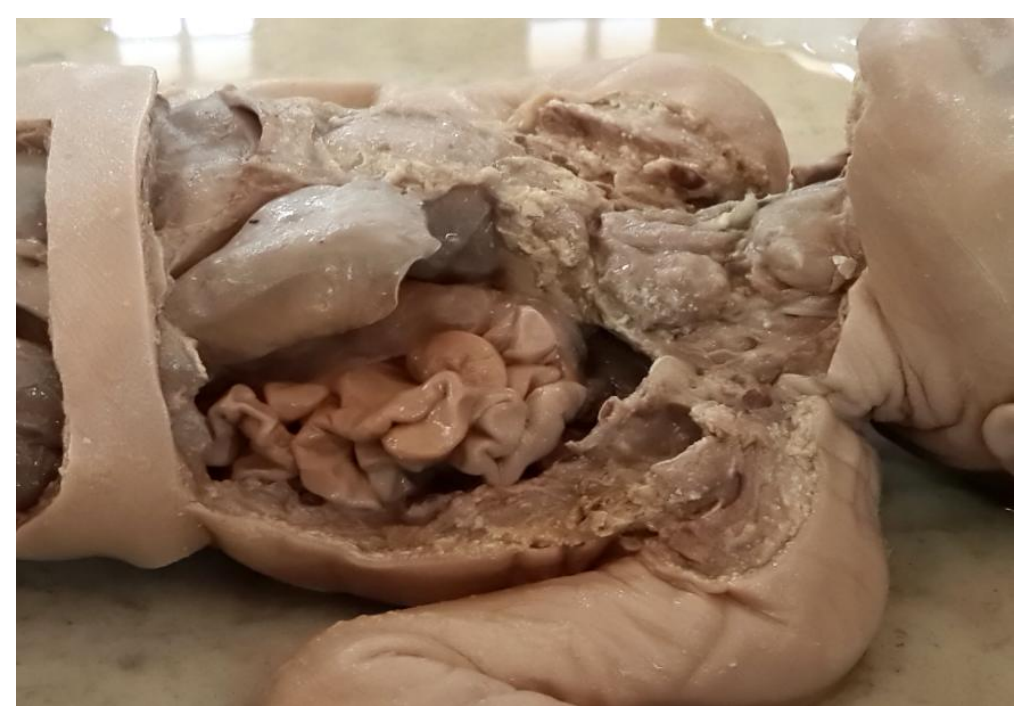

Figure 3 showing the intestinal loops in the left pleural cavity and mediastinal shift to the right side.

\section{Discussion:}

The diaphragm is a composite structure developing from four embryonic components which give rise to the adult derivatives:

1. Septum transversum forms the central tendon of diaphragm

2. Paired pleuroperitoneal membranes form the small peripheral part of diaphragm

3. Dorsal mesentery of esophagus form the crura of diaphragm

4. Mesoderm of the body wall form the large peripheral parts of diaphragm external to parts derived from pleuroperitoneal membranes.

$\mathrm{CDH}$ may occur when there are gaps in the diaphragm due to failure of closure of different components of diaphragm. It can be of four types:

1. Posterolateral hernia occurs due to Bochdalek's triangle, a triangular gap in the diaphragm which results due to failure of closure of pleuroperitoneal openings. These defects are usually unilateral and on the left side accounting for $80 \%$ cases of $\mathrm{CDH}^{4}$.

2. Retrosternal hernia occurs due to foramen of Morgagni, an abnormal large gap between the sternal and costal parts of the muscle.

3. Posterior hernia may occur due to the failure of development of the crura. 
4. Central hernia occurs rarely, when the entire half (usually the left) of diaphragm is absent.

Bilateral hernias are very rare accounting to only $2 \%$ of the cases ${ }^{5}$. Although CDH usually presents during neonatal period, $13 \%$ cases may be found in the later period ${ }^{6}$.

In our case, a large triangular opening or pleuroperitoneal hiatus was found on left side of the diaphragm. Usually the stomach is retained in the abdominal cavity in pleuroperitoneal hiatus hernia but in our case, whole of the stomach was herniated. A part of the right lobe of the liver was also herniated with the complete absence of spleen. There was severe hypoplasia of the left lung and apparent dextrocardia of heart.

Pulmonary hypoplasia and hypertension, the dysfunction of surfactant system combined with cardiac malformation may be the cause of respiratory distress and death. The presence of intestine in thoracic cavity during late fetal life causes malrotation and/or malfixation ${ }^{6}$ which further complicate the disease. Although the etiology of CDH is largely unknown, several genes on the long arm of chromosome 15 (15q) play a critical role in the development of the diaphragm ${ }^{8}$. The retinoic acid signaling pathway also has a key role ${ }^{9}$.

\section{Treatment:}

Since a large proportion of fetuses with $\mathrm{CDH}$ are diagnosed in utero by the routine prenatal ultrasound screening, it is advisable to direct the mother to a tertiary perinatal center where all the necessary obstetric, neonatal and surgical care can be taken ${ }^{10}$. Whenever diagnosed early, all efforts should be directed at enhancing antenatal lung growth like the use of Fetoscopic reversible tracheal obstruction. Surgical repair should be done only when the cardio-respiratory functions are stable after birth. Gentle ventilation with occasional ECMO (extracorporeal membrane oxygenation) helps in protecting the lungs during the intensive care postoperatively.

\section{Conclusion:}

$\mathrm{CDH}$ may be caused by disturbed molecular signaling during organogenesis. By ultrasonographic evaluation, the definitive diagnosis of the degree of displacement of abdominal organs into the thoracic cavity can be made. This will help in reducing the mortality as the prenatal management and postnatal surgical correction can be planned accordingly. The morbidity and mortality is high in CDH in spite of the recent advances. Since many aspects of the disease are still unknown, more research need to be done for the causation, prevention and treatment.

\section{References:}

[1] Adzick NS, Harrison MR, Glick PL, Nakayama DK, Manning FA, de Lorimier AA. Diaphragmatic hernia in fetus: prenatal diagnosis and outcome in 94 cases. J Pediatr Surg. 1985; 20: 357-361.

[2] Arvind Sehgal, Jagdish Chandra, Varinder Singh, A. K. Dutta and Deepak Bagga. Indian J Chest Dis Allied Sci 2002; 44: 57-60.

[3] Furuta Y, Nakamura Y, Miyamoto K. Bilateral congenital posterolateral diaphragmatic hernia. J Pediatr Surg. 1987; 22: 182-183.

[4] Clark RH, Hardin WD Jr, Hirschl RB, Jaksic T, Lally KP, Langham MR Jr, Wilson JM: Current surgical management of congenital diaphragmatic hernia: a report from the Congenital Diaphragmatic Hernia Study Group. J Pediatr Surg 1998, 33:1004-1009.

[5] Kufeji DI, Crabbe DC. Familial bilateral congenital diaphragmatic hernia. Pediatr Surg Int. 1999; 15: 58-60.

[6] Chetan G, Sreerag KS, Rathisharmila R, Barath J, Narayanan P, Jagdish S, Bhat VB. Unusual case of congenital diaphragmatic hernia - a case report. Curr Pediatr Res. 2008; 12: 5-7.

[7] Baoquan Q, Diez-Pardo JA, Tovar JA: Intestinal rotation in experimental congenital diaphragmatic hernia. J Pediatr Surg 1995, $30: 1457-1462$

[8] A. M. Holder, M. Klaassens, ${ }^{*}$ D. Tibboel, A. de Klein, B. Lee, and D. A. Scott. Genetic Factors in Congenital Diaphragmatic Hernia. Am J Hum Genet. May 2007; 80(5): 825-845.

[9] Tibboel R.Genetics in CDH, 2006; Presentation at Interdisciplinary Update on Congenital Diaphrag- matic Hernia, University of Heidelberg, Germany

[10] Shaw KS, Filiatrault D, Yazbeck S, St-Vil D: Improved survival for congenital diaphragmatic hernia, based on prenatal ultrasound diagnosis and referral to a combined obstetric-pediatric surgical center. J Pediatr Surg 1994, 29:1268-1269. 